\title{
Fatores de Risco Cardiovascular em Cardiologistas Especialistas pela Sociedade Brasileira de Cardiologia
}

\author{
Cardiovascular Risk Factors in Cardiology Specialists from the Brazilian Society of Cardiology
}

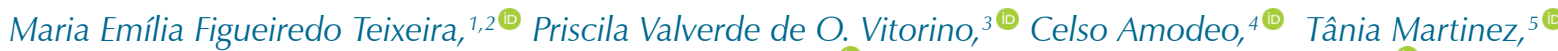 \\ Andréa Araujo Brandão, ${ }^{6}$ Eduardo Costa Duarte Barbosa, ${ }^{7}$ Audes Diógenes Magalhães Feitosa, ${ }^{8}{ }^{\circledR}$ Paulo Cesar B. \\ Veiga Jardim, ${ }^{\circledR 0}$ Ana Luiza Lima Souza, 1,2 Weimar Kunz Sebba Barroso, 1,2 \\ Universidade Federal de Goiás - Liga de Hipertensão Arterial, ${ }^{1}$ Goiânia, GO - Brasil \\ Universidade Federal de Goiás - Programa de Pós-Graduação em Ciências da Saúde, ${ }^{2}$ Goiânia, GO - Brasil \\ Pontifícia Universidade Católica de Goiás - Escola de Ciências Sociais e da Saúde, ${ }^{3}$ Goiânia, Goiás - Brasil \\ Escola paulista de Medicina da Universidade Federal de São Paulo (Unifesp EPM), ${ }^{4}$ São Paulo, SP - Brasil \\ Hospital do Coração (HCor) - Lipids, ${ }^{5}$ São Paulo, SP - Brasil \\ Universidade do Estado do Rio de Janeiro (UERJ) - Cardiologia, ${ }^{6}$ Rio de Janeiro, RJ - Brasil \\ Instituto de Cardiologia - Laboratório de Investigação Clínica (LIC), ${ }^{7}$ Porto Alegre, RS - Brasil \\ Laboratório de Imunopatologia Keizo Asami - Universidade Federal de Pernambuco - Clínica Médica, ${ }^{8}$ Recife, PE - Brasil \\ Universidade Federal de Goiás - Cardiologia, ${ }^{9}$ Goiânia, GO - Brasil
}

\section{Resumo}

Fundamento: Principal causa de morte em todo o mundo, as doenças cardiovasculares (DCV) e sua prevalência nos médicos cardiologistas são pouco conhecidas.

Objetivos: Descrever os hábitos de vida e os fatores de risco cardiovascular e verificar a prevalência de diagnóstico, conhecimento e controle dos fatores de risco cardiovasculares (FRCV) de médicos cardiologistas associados e especialistas pela Sociedade Brasileira de Cardiologia.

Métodos: Estudo multicêntrico nacional transversal que avaliou cardiologistas brasileiros por meio de questionário sobre hábitos de vida, doenças preexistentes, medicações em uso, medidas antropométricas, pressão arterial e dosagens de glicose e lípideos sanguíneos.

Resultados: Foram avaliados 555 cardiologistas, 67,9\% do sexo masculino, média de idade de 47,2 $\pm 11,7$ anos. A maioria era não tabagista $(\mathbf{8 8}, 7 \%)$, fisicamente ativa $(77,1 \%)$, consumia bebida alcóolica $(78,2 \%)$, com circunferência abdominal normal (51,7\%) e excesso de peso (56,1\%). As prevalências de hipertensão arterial sistêmica (HAS), diabetes mellitus (DM) e dislipidemia (DLP) foram de 32,4\%, 5,9\% e 49,7\%, respectivamente e, destes, apenas $57,2 \%, 45,5 \%$ e $49,6 \%$ sabiam ter as doenças.

Conclusões: Os cardiologistas brasileiros participantes do estudo apresentaram prevalências significativas de HAS, DM e DLP, mas apenas a metade dos participantes sabia ser portador dessas condições e, entre eles, as taxas de controle eram baixas para HAS e DLP, apesar de os cardiologistas serem profissionais detentores de conhecimento diferenciado sobre esses FRCV. Os achados representam um alerta para a abordagem dos FRCV em cardiologistas brasileiros e estimulam a realização de estudos futuros. (Arq Bras Cardiol. 2021; 116(4):774-781)

Palavras-chave: Doenças Cardiovasculares; Cardiologistas; Fatores de Risco; Antropometria; Hipertensão; Dislipidemias; Diabetes Mellitus; Estilo de Vida.

\footnotetext{
Abstract

Background: A major cause of death worldwide, cardiovascular diseases and their prevalence in cardiologists are little known.

Objectives: To describe life habits and cardiovascular risk factors (CVRF) and to investigate the prevalence of diagnosis, awareness, and control of these CVRF among cardiologists members affiliated to and specialists from the Brazilian Society of Cardiology.

Correspondência: Weimar Kunz Sebba Barroso •

Universidade Federal de Goiás - Liga de Hipertensão Arterial - Av. Universitária Hospital das Clinicas. CEP 74605-220, Goiânia, GO - Brasil E-mail: sebbabarroso@gmail.com

Artigo recebido em 20/02/2020, revisado em 28/08/2020, aceito em 09/09/2020
}

DOI: https://doi.org/10.36660/abc.20200125 
Methods: National multicenter cross-sectional study to assess Brazilian cardiologists using a questionnaire on life habits, preexisting diseases, current medications, anthropometric measurements, blood pressure, and levels of glucose and lipids.

Results: A total of 555 cardiologists were evaluated, of which $67.9 \%$ were male, with a mean age of $47.2 \pm 11.7$ years. Most were non-smoker (88.7\%) and physically active (77.1\%), consumed alcohol (78.2\%), had normal weight circumference (51.7\%), and were overweight (56.1\%). The prevalence of systemic arterial hypertension (SAH), diabetes mellitus (DM), and dyslipidemia (DLP) were 32.4\%, $5.9 \%$, and $49.7 \%$, respectively, of which only $57.2 \%, 45.5 \%$, and $49.6 \%$, respectively, were aware of the diseases.

Conclusions: The Brazilian cardiologists participating in the study had a high prevalence of SAH, DM and DLP, but only a half of participants were aware of these conditions and, among these, the rates of controlled disease were low for SAH and DLP, although cardiologists are professionals with great knowledge about these CVRF. These findings represent a warning sign for the approach of CVRF in Brazilian cardiologists and encourage the conduction of future studies. (Arq Bras Cardiol. 2021; 116(4):774-781)

Keywords: Cardiovascular Diseases; Cardiologists; Risk Factors; Antropometry; Hypertension; Dsylipidemias; Diabetes Mellitus; Life Style.

Full texts in English - http://www.arquivosonline.com.br

\section{Introdução}

Dentre os fatores de risco cardiovascular (FRCV), aqueles com maior impacto no aumento das taxas de morbidade e mortalidade são hipertensão arterial sistêmica (HAS), diabetes mellitus (DM) dislipidemia (DLP) e tabagismo. ${ }^{1}$ Além disso, os hábitos de vida desfavoráveis levam ao excesso de peso e, juntos, interferem de maneira significativa na prevalência desses fatores, ${ }^{2}$ com consequente aumento da incidência de desfechos cardiovasculares, tais como morte súbita, acidente vascular encefálico (AVE), infarto agudo do miocárdio (IAM), insuficiência cardíaca, doença arterial periférica e doença renal crônica. ${ }^{3-5}$

Os profissionais de saúde, incluindo a classe médica, , especialmente o cardiologista, assumem papel fundamentalcomo responsáveis por diagnosticar e tratar doenças cardiovasculares. ${ }^{6}$ Além disso, o cardiologista brasileiro é visto, com frequência, como o responsável pelos cuidados com a saúde global do paciente adulto. ${ }^{7}$ Portanto, é de se esperar que, além de cuidar, os cardiologistas também sirvam de modelo a ser seguido e, principalmente, que assumam uma postura pessoal de hábitos de vida saudáveis. ${ }^{8}$

Poucos são os estudos que avaliaram o risco cardiovascular e os hábitos de vida de cardiologistas brasileiros; ${ }^{9}$ portanto, foram objetivos deste estudo: (1) verificar hábitos de vida e FRCV e (2) identificar as prevalências de diagnóstico, conhecimento e controle de HAS, DM e DLP em médicos cardiologistas associados e especialistas pela Sociedade Brasileira de Cardiologia (SBC).

\section{Métodos}

Tipo de estudo, população, amostra e critérios de inclusão

\section{Estudo multicêntrico nacional transversal descritivo.}

Em 2017, o Brasil possuía 451.777 médicos, com aproximadamente 25.000 (5,5\%) cardiologistas; ${ }^{10}$ destes, 11.495 tinham o título de especialista em Cardiologia (TEC). ${ }^{11}$ A população de referência foi constituída por 14.201 médicos cardiologistas sócios da SBC em 2017, distribuídos em todo o território nacional, com sociedades estaduais em 24 unidades federativas. Optou-se por realizar a pesquisa com cardiologistas portadores do TEC/
SBC para uniformização da amostra em relação ao nível de conhecimento científico.

A amostra foi de conveniência, tendo sido incluídos no estudo 555 médicos com o TEC/SBC e membros ativos da SBC, o que representa $4,8 \%$ da população de referência.

\section{Locais de realização e coordenação do estudo}

Todos os 24 representantes regionais da SBC/Diretoria de Prevenção em Saúde Cardiovascular (FUNCOR) foram convidados a integrar o grupo de pesquisadores deste projeto. Desses, 15 aceitaram o convite e, juntamente com outros três centros convidados [Instituto Dante Pazzanese de Cardiologia (IDPC), Liga de Hipertensão Arterial da Universidade Federal de Goiás (LHA/UFG) e Unidade de Hipertensão da Universidade Estadual do Rio de Janeiro], totalizaram 18 centros de pesquisa que efetivamente integraram o grupo de investigadores e coinvestigadores que coletaram dados no período de maio até outubro de 2017.

A coleta de dados foi feita nas nos seguintes estados: Bahia, Distrito Federal, Goiás, Mato Grosso, Mato Grosso do Sul, Minas Gerais, Pará, Paraíba, Paraná, Pernambuco, Rio de Janeiro, Rio Grande do Norte, Rio Grande do Sul, Rondônia, São Paulo e Tocantins.

Todo o trabalho foi coordenado pela Diretoria da SBC/ FUNCOR, juntamente com as instituições universitárias IDPC e LHA/UFG.

\section{Procedimentos do estudo}

Foram realizadas reuniões presenciais com todos os investigadores em maio e junho de 2017 para discussão sobre o desenho do estudo e a coleta de dados. Cada investigador, após treinamento, capacitou sua equipe local para o seguimento rigoroso dos procedimentos do estudo. A coleta foi feita pelo próprio pesquisador médico responsável, ou por outros cardiologistas ou estudantes de medicina devidamente treinados.

Os participantes do estudo receberam explicações acerca do objetivo do estudo, forma de coleta dos dados avaliados e sobre o termo de consentimento livre e esclarecido (TCLE), que foi lido e assinado por todos antes do início de qualquer procedimento do estudo. 
A entrevista foi realizada individualmente em ambiente privativo e em horário e local previamente acordados com os participantes. O formulário de entrevista continha perguntas referentes a dados pessoais, hábitos de vida e antecedentes pessoais de doenças. Também foram realizadas medidas antropométricas e de pressão arterial (PA) e exames de glicemia e perfil lipídico.

A idade foi calculada a partir da data de nascimento. O sexo foi categorizado em masculino e feminino. Os hábitos de vida avaliados foram tabagismo ( $\mathrm{sim} /$ não); consumo de bebidas alcóolicas (não/sim, para qualquer quantidade de consumo) e prática de atividade física ( $\operatorname{sim} /$ não e tempo semanal, sendo considerado ativo o praticante de pelo menos 150 minutos semanais). ${ }^{12}$

As variáveis antropométricas coletadas foram altura, peso e circunferência da cintura. A altura foi referida pelo participante; ${ }^{13}$ o peso foi aferido com utilização de balança digital OMRON HN-290T, após retirada de acessórios e calçados e com uso de roupas leves. ${ }^{14}$

O IMC foi calculado por meio da fórmula peso/ altura $^{15}$ e classificado em: baixo peso $\left(<18,5 \mathrm{~kg} / \mathrm{m}^{2}\right)$, eutrófico (18,5-24,9 kg/m²); sobrepreso (25-29,9 kg/m²); obesidade 1 (30-34,9 kg/m²), obesidade 2 (35-39,9 kg/ $\left.\mathrm{m}^{2}\right)$ e obesidade $3\left(\geq 40 \mathrm{~kg} / \mathrm{m}^{2}\right) .^{16}$

A circunferência da cintura foi medida com fita inextensível ${ }^{14}$ e considerada alterada se maior que 88 $\mathrm{cm}$ para mulheres e maior que $102 \mathrm{~cm}$ para homens. ${ }^{17}$

A aferição da PA foi realizada com esfigmomanômetro automático da marca OMRON, modelo HBP $1100 . .^{18-20}$ de acordo com a recomendação da 7a Diretriz Brasileira de Hipertensão Arterial. ${ }^{21}$ Foram obtidas três aferições da PA, excluída a primeira medida e calculada a média das duas seguintes. Os participantes foram classificados a partir do valor da média pressórica em normotensos (PA $\leq$ 120/80 mmHg), pré-hipertensos (121-139/81-89 mmHg), ou hipertensos estágio 1 (140-159/90-99 mmHg), 2 (160179/100-109 mmHg) ou 3 (PA $\geq 180 / 110 \mathrm{mmHg}$ ). ${ }^{21}$

A glicemia e os lipídeos séricos foram obtidos com os aparelhos On Call Plus e Mission Cholesterol, respectivamente. Todos os valores dos exames foram obtidos diretamente dos aparelhos em $\mathrm{mg} / \mathrm{dL}$, exceto o LDL, que foi calculado pela fórmula de Friedewald. ${ }^{22}$

Foram realizadas dosagens sem o jejum e, portanto, considerados os valores de glicemia alterados $\geq 160 \mathrm{mg} /$ $\mathrm{dL}^{23}$ e portadores de DLP aqueles com LDL $\geq 130 \mathrm{mg} / \mathrm{dL}$ e/ou triglicérides $\geq 175 \mathrm{mg} / \mathrm{dL} .{ }^{24}$

Para o diagnóstico de HAS, DM e DLP, foi considerado pelo menos um dos seguintes critérios: relato de ser portador, feito pelo próprio participante e/ou uso de anti-hipertensivos e/ou PA $\geq 140 \times 90 \mathrm{mmHg}$ na média das medidas casuais; uso de hipoglicemiantes orais e/ou de insulina e/ou glicemia capilar ocasional $\geq 200 \mathrm{mg} / \mathrm{dL}$; uso de estatinas, fibratos, ezetimiba e/ou triglicérides $\geq$ $175 \mathrm{mg} / \mathrm{dL}$ e/ou LDL $\geq 130 \mathrm{mg} / \mathrm{dL}$.

Foi considerado conhecimento da doença o relato do próprio médico sobre ser portador. Os dados referentes às frequências de HAS, DM e DLP foram analisados em relação aos obtidos na Pesquisa Nacional de Saúde (PNS) ${ }^{25}$ e no sistema de vigilância de fatores de risco para doenças crônicas não transmissíveis por inquérito telefônico (VIGITEL); ${ }^{26}$ para essa análise, foi considerado somente o autorrelato do participante (dados referidos).

Foram consideradas controladas a HAS com pressão arterial sistólica $<140 \mathrm{mmHg}$ e pressão arterial diastólica $<90$ mmHg, DM com glicemia < 200 mg/dL e DLP com $\mathrm{LDL}<130$ e triglicérides $<175 \mathrm{mg} / \mathrm{dL} .{ }^{21,23,24}$

\section{Análise estatística}

Os dados foram digitados no programa Excel para Mac versão 16.30 e analisados em software de análise estatística Stata, versão 14. Foi realizada estatística descritiva com apresentação de médias, desvio padrão e frequências absoluta e relativa.

\section{Aspectos éticos}

O projeto, desenvolvido pela FUNCOR da SBC, gestão 2016/2017, foi aprovado pelo Comitê de Ética em Pesquisa (CEP) do IDPC, sob o número 2.016.859. Todos os participantes assinaram o TCLE antes de qualquer procedimento do estudo, que seguiu a Resolução 466/2012.

\section{Resultados}

Foram avaliados 555 cardiologistas com idade média de 47,2 $\pm 11,7$ anos, sendo 159 (28,6\%) da Região CentroOeste, 147 (26,5\%) da Região Nordeste, 103 (18,6\%) da Região Norte, 103 (18,6\%) da Região Sudeste e 43 (7,7\%) da Região Sul.

A maioria dos participantes da pesquisa era do sexo masculino, ativo, com tempo médio de atividade física de 200,0 $\pm 106,8$ minutos por semana, não fumante, e fazia uso de bebida alcóolica (Tabela 1).

De acordo com as medidas obtidas durante a entrevista, a maioria dos médicos apresentou níveis pressóricos na categoria de pré-hipertensão e valores de glicemia, LDL e triglicérides dentro da normalidade (Tabela 2).

A prevalência de HAS foi de $32,4 \%(n=180)$; destes, $57,2 \%(n=103)$ conheciam essa condição, e 48,3\% ( $n=87)$ estavam com a pressão controlada. A prevalência de DM foi de $5,9 \%(n=33)$; destes, $45,5 \%(n=15)$ sabiam que tinham a doença, e 78,8\% $(n=26)$ estavam com a glicemia dentro dos valores de normalidade. A dislipidemia apresentou valores de prevalência, conhecimento e controle de $49,7 \%(n=276), 49,6 \%(n=137)$ e $31,1 \%$ $(n=86)$, respectivamente (Figura 1$)$.

Em relação aos desfechos cardiovasculares, 4 (0,72\%) cardiologistas referiram já ter apresentado IAM, e 1 $(0,18 \%)$ referiu AVE. Todos os quatro portadores de doença arterial coronária diagnosticada estavam em uso de algum anti-agregante plaquetário.

A Tabela 3 apresenta as frequências dos FRCV e desfechos cardiovasculares do PNS, ${ }^{25}$ do VIGITEL ${ }^{26}$ e os achados do presente estudo, considerando somente as doenças que foram autorreferidas. 
Tabela 1 - Descrição da amostra segundo o sexo, estilo de vida e condições gerais de saúde, $n=555,2017$

\begin{tabular}{|c|c|}
\hline Variável & n (\%) \\
\hline \multicolumn{2}{|l|}{ Sexo } \\
\hline Feminino & $178(32,1)$ \\
\hline Masculino & $377(67,9)$ \\
\hline \multicolumn{2}{|l|}{ Faixa étária } \\
\hline$<40$ anos & $183(33,2)$ \\
\hline$\geq 40$ anos & $368(66,8)$ \\
\hline \multicolumn{2}{|l|}{ Tabagismo } \\
\hline Sim & $03(0,5)$ \\
\hline Não & $492(88,7)$ \\
\hline Ex-tabagista & $60(10,8)$ \\
\hline \multicolumn{2}{|l|}{ Sedentarismo } \\
\hline Sim & $127(22,9)$ \\
\hline Não & $428(77,1)$ \\
\hline \multicolumn{2}{|c|}{ Consumo de bebidas alcoólicas } \\
\hline Sim & $434(78,2)$ \\
\hline Não & $121(21,8)$ \\
\hline \multicolumn{2}{|c|}{ Circunferência abdominal } \\
\hline Normal & $285(51,7)$ \\
\hline Elevada & $266(48,3)$ \\
\hline \multicolumn{2}{|c|}{ Classificação segundo o índice de massa corporal } \\
\hline Sem excesso de peso & $243(43,9)$ \\
\hline Sobrepeso & $232(41,9)$ \\
\hline Obesidade & $79(14,2)$ \\
\hline
\end{tabular}

\section{Discussão}

Este é o primeiro estudo no Brasil a avaliar cardiologistas com TEC das cinco regiões geográficas para a presença de FRCV e hábitos de vida. Os cardiologistas apresentaram prevalências mais baixas de sedentarismo e tabagismo e mais altas de etilismo em relação a estudos que avaliaram a população em geral, como o $\mathrm{PNS}^{25}$ e o VIGITEL, ${ }^{26}$ e também prevalência maior de DLP, ligeiramente menor de HAS, e mais baixa de DM. Entretanto, as taxas de conhecimento de HAS, DM e DLP e as taxas de controle da HAS e DLP observadas foram baixas, considerando-se que a população estudada é de cardiologistas, conhecedores da importância do controle dos FRCV.

Na população brasileira, a prevalência de HAS varia de $30 \%$ a $36 \% ;{ }^{27,28}$ a de DM é $11,4 \% ;{ }^{29}$ e a DLP se divide em hipercolesterolemia, com prevalência de aproximadamente $45,5 \%,{ }^{30}$ e hipertrigliceridemia, com prevalência na América Latina de $26,5 \%$ a $31,2 \% .^{31,32}$ Além disso, a prevalência de excesso de peso (sobrepeso/obesidade) no Brasil é de $57 \%$ em homens e $43 \%$ em mulheres. ${ }^{33}$ No grupo aqui estudado, considerando os dados referidos e aferidos, houve diagnóstico de $32,4 \%$ de HAS, 4, 9\% de DM, 51,7\% de DLP (hipercolesterolemia e/ou hipertrigliceridemia), e $56 \%$ de excesso de peso $(67,1 \%$ entre os homens e $32,2 \%$ entre as mulheres).
Tabela 2 - Classificação dos cardiologistas de acordo com a medida da pressão arterial, glicemia casual e lipídeos séricos, 2017

\begin{tabular}{lc}
\hline Classificação & $\mathbf{n}(\%)$ \\
\hline Pressão arterial (n=555) & $204(36,8)$ \\
\hline Normotensos & $264(47,6)$ \\
\hline Pré-hipertensos & $75(13,5)$ \\
\hline Hipertensão I & $08(1,4)$ \\
\hline Hipertensão II & $04(0,7)$ \\
\hline Hipertensão III & \\
\hline Glicemia casual (n=555) & $548(98,7)$ \\
\hline Não alterada & $07(1,3)$ \\
\hline Alterada & $411(76,4)$ \\
\hline LDL (n=538) & $127(23,6)$ \\
\hline Não alterado & \\
\hline Elevado & $463(84,6)$ \\
\hline Triglicérides ( $\mathbf{n}=547)$ & $84(15,4)$ \\
\hline Não alterado &
\end{tabular}

A falta de conhecimento de ser portador desses FRCV é sabidamente alta na população em geral, mas chama a atenção que também seja elevada entre os cardiologistas, levando-nos a considerar que haja uma negligência desses profissionais em relação aos cuidados com a própria saúde. Essa demora no conhecimento, diagnóstico precoce e tratamento adequado pode aumentar o risco de desfechos relacionados. ${ }^{34}$

Sabe-se que a educação em saúde para população leiga é capaz de gerar melhora dos hábitos de vida, impactando em redução das doenças cardiovasculares. ${ }^{35}$ Por esse motivo, surgiu o questionamento sobre a qualidade do autocuidado dos médicos cardiologistas, como portadores desse conhecimento específico. Estudantes de medicina avaliados para FRCV apresentaram prevalência semelhante à população geral de mesma faixa etária, exceto por maior sedentarismo e IMC, suscitando assim o debate sobre a carga horária elevada do curso, a qual pode influenciar na pequena disponibilidade de tempo para a prática de hábitos de vida saudáveis, em comparação com outros adultos jovens. ${ }^{36}$ Em outro grupo de estudantes de medicina, foram vistos níveis mais baixos de obesidade em comparação com a população da mesma idade, e níveis melhores de lipídeos séricos, mas um elevado consumo de fast food e bebidas alcoólicas, assim como maior sedentarismo, o que também pode ter como explicação a pequena disponibilidade de tempo e o elevado nível de estresse relacionado ao curso. ${ }^{37}$

Sabe-se também que, muitas vezes, a rotina de trabalho pode afetar negativamente a adoção de práticas de saúde e bem-estar, mesmo que o profissional seja detentor do conhecimento acerca do assunto, como são os profissionais da área de saúde..$^{38} \mathrm{O}$ trabalho nessa área exige a presença de equipes noturnas e, com frequência, esses profissionais trabalham em mais de um emprego. 


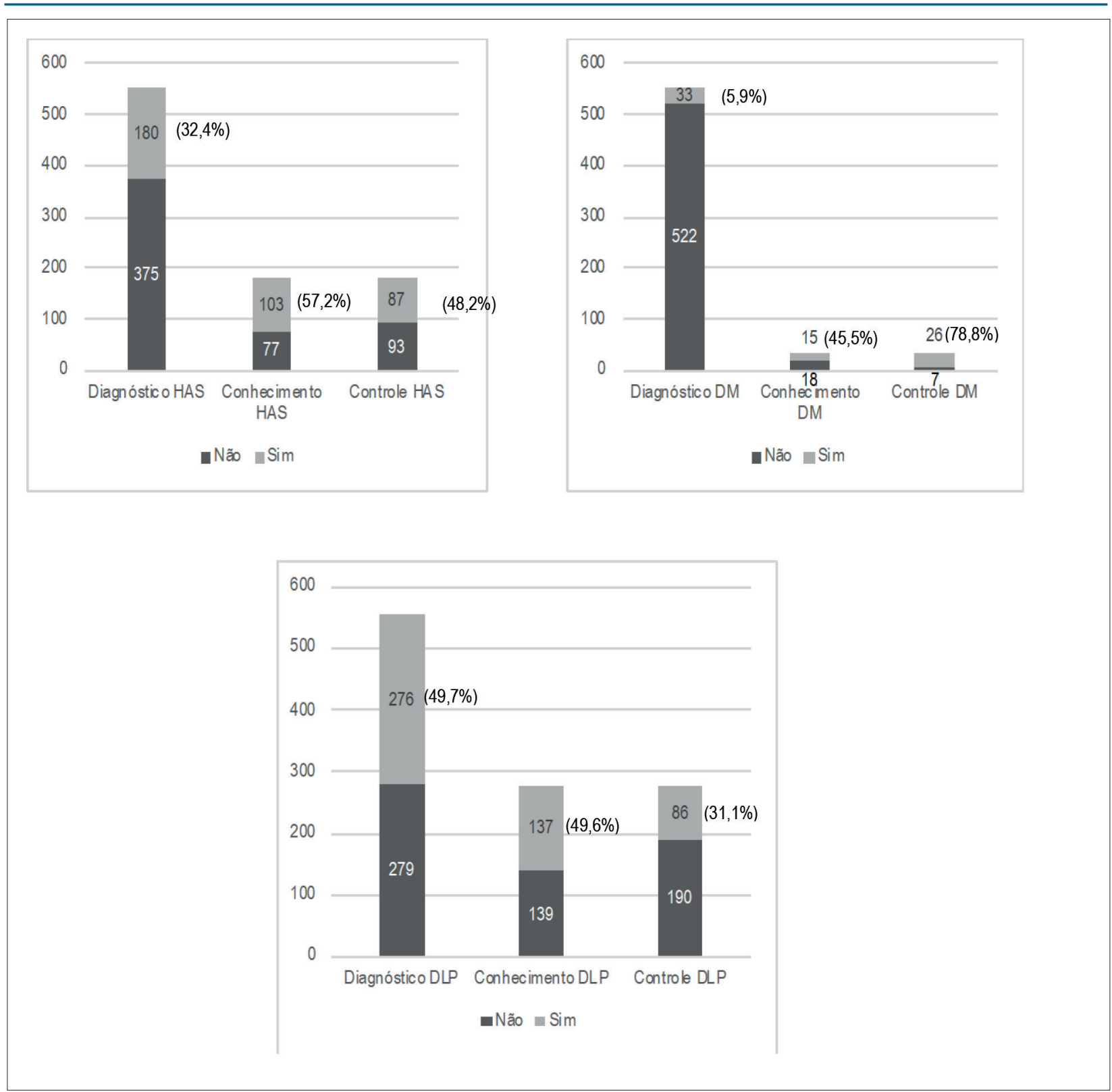

Figura 1 - Prevalência de diagnóstico, conhecimento e controle de HAS, DM e DLP em cardiologistas, $n=555$, 2017. DLP: dislipidemia; DM: diabetes mellitus; HAS: hipertensão arterial sistêmica.

Tabela 3 - Prevalência de fatores de risco e desfechos cardiovasculares na população geral e entre os cardiologistas. $n=555,2017$

\begin{tabular}{lcccc}
\hline & PNS & Vigitel & Cardiologistas (referido) & Cardiologistas (aferido) \\
\hline Sedentarismo & 46 & 61,9 & 22,9 & - \\
\hline Etilismo & 24 & 17,9 & 78,2 & - \\
\hline Tabagismo & 15 & 9,3 & 0,5 & - \\
\hline Hipertensão arterial & 21,4 & 24,7 & 18,6 & 32,4 \\
\hline Diabetes mellitus & 6,2 & 7,7 & 2,7 & 5,9 \\
\hline Dislipidemia & 12,5 & - & 24,7 & 49,7 \\
\hline Infarto agudo do miocárdio & 4,2 & - & 0,7 & - \\
\hline Acidente vascular encefálico & 1,5 & - & 0,2 & - \\
\hline
\end{tabular}

Fonte: PNS, ${ }^{25}$ Vigitel $2018^{26}$. PNS: Pesquisa Nacional de Saúde. 
Dessa forma, dificilmente conseguem praticar exercícios físicos regularmente ou priorizar alimentos equilibrados do ponto de vista nutricional.

Por outro lado, a mesma discussão pode ser levantada sem a necessidade de enfatizar o trabalho noturno como malefício mais importante, mas considerando-se apenas a carga horária excessiva desses profissionais, independente do horário. Dois grupos diferentes avaliaram seus profissionais quanto à prevalência dos FRCV, incluindo toda a equipe multiprofissional na avaliação. Em um hospital geral, foi encontrada prevalência elevada de FRCV em todas as classes profissionais avaliadas. ${ }^{39}$ Resultados semelhantes foram encontrados em outro grupo, com uma situação ainda mais preocupante, que é a falta de conhecimento dessas pessoas acerca do seu quadro de saúde já alterado. ${ }^{40}$

Nos subgrupos de médicos cardiologistas versus não cardiologistas, não foram observadas diferenças significativas em relação aos níveis séricos de colesterol e frações, assim como em relação ao escore de risco de Framingham, mas os cardiologistas ingeriam mais bebidas alcoólicas, e ambos grupos estavam com IMC acima do ideal, em média. ${ }^{41}$

Em análise comparativa com os inquéritos populacionais $\mathrm{PNS}^{25}$ e VIGITEL, ${ }^{26}$ os cardiologistas avaliados no presente estudo referem menos tabagismo e sedentarismo, mas ingerem mais bebida alcoólica. Além disso, considerando apenas os FRCV referidos, relataram menos HAS e DM, porém mais DLP. Esses dados preocupam, não apenas pela falta de conhecimento, mas também porque colocam a questão da credibilidade de inquéritos que utilizam apenas dados referidos.

Sabe-se que HAS, DM e DLP ${ }^{42}$ resultam de fatores como genética e envelhecimento (não modificáveis), mas também têm relação com hábitos de vida e, nesse contexto, é de se esperar que indivíduos com melhor conhecimento em relação aos riscos cardiovasculares tenham hábitos mais saudáveis, ${ }^{43-45}$ Amplamente conhecedores do assunto, esperava-se que os médicos cardiologistas praticassem bons hábitos em sua totalidade, de modo a prevenir tais doenças, fato contradito em nossa amostra em relação ao consumo de bebidas alcoólicas, mas confirmado em relação ao tabagismo e à atividade física. Da mesma forma, encontramos prevalências semelhantes ou mesmo mais elevadas dos principais FRCV quando comparados à população, exceto em relação a DM.

Por fim, o percentual relatado de IAM $(0,72 \%)$ e de $\operatorname{AVE}(0,18 \%)$ na amostra é bem menor que o da população em geral, o que pode estar relacionado ao uso regular e frequente de medicamentos, por conhecimento do tratamento adequado e facilidade do acesso a eles. Além disso, a idade média do grupo é baixa (47,2 anos) e pode justificar, em parte, a prevalência baixa dos desfechos IAM e AVE. ${ }^{46}$

O presente estudo apresenta como limitações: a ausência do HDL na avaliação de DLP, devido a uma limitação de análise do aparelho; não aplicação de instrumentos para avaliação de atividade física e do consumo de álcool, fato que pode ter superestimado essas taxas; e não foram obtidos exames de bioquímica em jejum. Vale ressaltar, contudo, que foram utilizados aparelhos iguais para aferições tanto de antropometria quanto da PA e da bioquímica sanguínea, com treinamento prévio dos coinvestigadores e coordenação geral dos centros de referência, demonstrando padronização adequada de procedimento.

Destacamos ainda que a amostra não foi representativa dos cardiologistas da SBC, pois se trata de uma amostra de conveniência, fato que pode relativizar os resultados e as discussões apresentadas. Entretanto, foram avaliados cardiologistas em todo o território nacional e, portanto, esse estudo representa um alerta para a abordagem das condições identificadas e para a realização de estudos futuros em cardiologistas brasileiros.

\section{Conclusão}

A maioria dos cardiologistas era do sexo masculino, ativo, não fumante; fazia uso de bebida alcóolica; e apresentava prevalências significativas de HAS, DM e DLP, próximas às de outros levantamentos em populações brasileiras. Entretanto, embora tenham conhecimento sobre esses FRCV, aproximadamente a metade sabia ser portador dessas condições e estava com a pressão controlada; além disso, um terço estava com os lipídeos dentro dos valores de normalidade, mas a maioria estava com a glicemia controlada. Os achados representam alerta para adequada abordagem dos FRCV em cardiologistas brasileiros e apontam para a necessidade de estudos futuros.

\section{Coinvestigadores}

Alberto de Almeida Las Casas Júnior (Goiás), Alexandre Jorge de Andrade Negri (Paraíba), Andrés Gustavo Sánchez Esteva (Tocantins), Antônio Carlos Avanza Junior (Espírito Santo), Christiano Henrique Souza Pereira (Mato Grosso do Sul), Claudine Maria Alves Feio (Pará), Daniela Martins Lessa Barreto (Alagoas), Diana Patrícia Lamprea Sepúlveda (Pernambuco), Érika Maria Gonçalves Campana (Rio de Janeiro), Evandro Guimarães de Souza (Minas Gerais), Ezilaine Nascimento Rosa (Mato Grosso), Fátima Elizabeth Fonseca de Oliveira Negri (Paraíba), Harry Corrêa Filho (Santa Catarina), João Paulo Fernandes Caixeta (Goiás), João Roberto Gemelli (Rondônia), Joilma Silva Prazeres Tobias (Maranhão), José Fernando Vilela Martin (São Paulo), Juan Carlos Yugar Toledo (São Paulo), Lara Araújo Dias (Goiás), Maurício Pimentel (Rio Grande do Sul), Nivaldo Menezes Filgueiras Filho (Bahia), Sandra Andrade Mendonça Hilgemberg (Rio Grande do Norte), Sílvio Henrique Barberato (Paraná), Simone Nascimento dos Santos (Distrito Federal), Thaynara de Moraes Pacheco (Goiás).

\section{Contribuiç̧ão dos autores}

Concepção e desenho da pesquisa: Amodeo C, Martinez T, Brandão AA, Barroso WKS; Obtenção de dados: Teixeira MEF, Vitorino PVO, Barroso WKS; Análise e interpretação dos dados: Teixeira MEF, Vitorino PVO, Amodeo C, Martinez T, Brandão AA, Barbosa ECD, Feitosa ADM, Barroso WKS; Análise estatística: Teixeira MEF, Vitorino PVO, Souza ALL, Barroso WKS; Obtenção de financiamento: Amodeo C, 
Martinez T, Barroso WKS; Redação do manuscrito: Teixeira MEF, Vitorino PVO, Brandão AA, Barbosa ECD, Feitosa ADM, Jardim PCBV, Souza ALL, Barroso WKS; Revisão crítica do manuscrito quanto ao conteúdo intelectual: Teixeira MEF, Vitorino PVO, Amodeo C, Martinez T, Brandão AA, Barbosa ECD, Feitosa ADM, Jardim PCBV, Souza ALL, Barroso WKS.

\section{Potencial conflito de interesses}

Declaro não haver conflito de interesses pertinentes.

\section{Referências}

1. Vos T, Abajobir AA, Abate KH, Abbafati C, Abbas KM, Abd-Allah F, et al. Global, regional, and national incidence, prevalence, and years lived with disability for 328 diseases and injuries for 195 countries, 1990-2016: a systematic analysis for the Global Burden of Disease Study 2016. Lancet. 2017 Sep 16;390(10100):1211-59.

2. Masana L, Ros E, Sudano I, Angoulvant D, Ibarretxe Gerediaga D, Murga Eizagaechevarria $\mathrm{N}$, et al. Is there a role for lifestyle changes in cardiovascular prevention? What, when and how. Atheroscler Suppl. 2017 Apr; 26: 2-15.

3. Lewington S, Clarke R, Qizilbash N, Peto R, Collins R. Age-specific relevance of usual blood pressure to vascular mortality: a meta-analysis of individual data for one million adults in 61 prospective studies. Lancet. 2002 Dec 14;360(9349):1903-13.

4. Weber MA, Schiffrin EL, White WB, Mann S, Lindholm LH, Kenerson JG, et al. Clinical practice guidelines for the management of hypertension in the community a statement by the American Society of Hypertension and the International Society of Hypertension. J Hypertens. 2014 Jan;32(1):3-15.

5. Williams B, Mancia G, Spiering W, Agabiti Rosei E, Azizi M, Burnier M, et al. 2018 ESC/ESH Guidelines for the management of arterial hypertension. Eur HeartJ. 2018 Sep 1;39(33):3021-104.

6. Ribeiro RQ. Cardiologista: um Prometeu acorrentado. Arq Bras Cardiol. 2010 Jul;95(1):e24-5.

7. Mesquita ET, Correia ETO, Barbetta LMDS. Profile of Brazilian Cardiologists: An Overview of Female Leadership in Cardiology and Stress - Challenges for the Next Decade. Arq Bras Cardiol. 201908 8;113(1):69-70.

8. Jardim TV, Sousa AL, Povoa TI, Barroso WK, Chinem B, Jardim L, et al. The natural history of cardiovascular risk factors in health professionals: 20-year follow-up. BMC Public Health. 2015 Nov 11;15:1111.

9. Dioguardi G, Pimenta J, Knoplich J, Ghorayeb N, Ramos LR, Giannin SD. Fatores de risco para doenças cardiovasculares em médicos: dados preliminares do projeto vidam da associação paulista de medicina. Arq . Bras. Cardiol. 1994 Jul;62:383-8.

10. Scheffer M, Biancarelli A, Cassenote A. Demografia médica no Brasil 2015. [Internet]. São Paulo: Universidade de São Paulo; Conselho Regional de Medicina do Estado de São Paulo; Conselho Federal de Medicina; 2015. 285p. [citado 30 Jun 2017]. Disponível em: http://www.usp.br/agen/wpcontent/uploads/DemografiaMedica30nov20153.pdf

11. Sociedade Brasileira de Cardiologia. CJTEC - Título de Especialista em Cardiologia [Internet]. 2018 [citado 15 de novembro de 2019]. Disponível em: http://educacao.cardiol.br/cjtec/especialistas.asp

12. World Health Organization. Global recommendations on physical activity for health. World Health Organization; 2010.

13. Peixoto MR, Benício MH, Jardim PC. [Validity of self-reported weight and height: the Goiânia study, Brazil]. Rev Saude Publica. 2006 Dec;40(6):1065-72.

14. Lohman TG, Roche AF, Martorell R. Anthropometric standardization reference manual. Champaign: Human Kinetics; 1988).

\section{Fontes de financiamento}

O presente estudo foi parcialmente financiado pela Indústria Farmacêutica EMS e de equipamentos Medlevesson e Omron.

\section{Vinculação acadêmica}

Este artigo é parte de dissertação de Mestrado de Maria Emilia Figueiredo Teixeira pela Pós-graduação em Ciências da Saúde - UFG.

15. Ross WD, DrinkwaterDT, BaileyDA, Marshall GR, Leahy RM. Kinanthropometry: traditions and new perspectives. In: Ostyn M, Beunen G, Simons J, editors. Kinanthropometry II. Baltimore: University Park Press, 1980:3-26.

16. Calle EE, Thun MJ, Petrelli JM, Rodriguez C, Heath CW. Body-mass index and mortality in a prospective cohort of U.S. adults. N Engl J Med. 1999 Oct 7;341(15):1097-105.

17. World Health Organization. Waist circumference and waist-hip ratio: report of a WHO expert consultation. Geneva: World Health Organization; 2008.

18. Dab ${ }^{\circledR}$ Educational Trust Limited. Comparison of the Omron HBP-1100 with the Omron HBP-1300 Devices [Internet]. 2014 [citado 15 de novembro de 2019]. Available at: www.dableducational.orgFormDET6140526

19. Cao X, Song C, Guo L, Yang J, DengS, XuY, etal. Quality Control and Validation of Oscillometric Blood Pressure Measurements Taken During an Epidemiological Investigation. Medicine (Baltimore). 2015 Sep;94(37):e1475.

20. Meng L, Zhao D, Pan Y, Ding W, Wei Q, Li H, et al. Validation of Omron HBP1300 professional blood pressure monitor based on auscultation in children and adults. BMC Cardiovasc Disord. 2016 Jan 13;16:9.

21. Malachias MV. 7th Brazilian Guideline of Arterial Hypertension: Presentatio Arq Bras Cardiol. 2016 09;107(3 Suppl 3):0.

22. Friedewald WT, Levy RI, Fredrickson DS. Estimation of the concentration of low-density lipoprotein cholesterol in plasma, without use of the preparative ultracentrifuge. Clin Chem. 1972 Jun;18(6):499-502.

23. Sociedade Brasileira de Diabetes. Diretrizes da Sociedade Brasileira de Diabetes (2017-2018). São Paulo: Editora Clannad; 2017.

24. Faludi A, IzarM, Saraiva J, Chacra A, Bianco H, Afiune Neto A, et al. Atualização da Diretriz Brasileira de dislipidemias e prevenção da aterosclerose - 2017. Arq Bras Cardiol. 2017;109(1).

25. Instituto Brasileiro de Geografia e Estatística. Pesquisa Nacional de Saúde 2013 percepção do estado de saúde, estilos de vida e doenças crônicas - Brasil, Grandes Regiões e Unidades da Federação [Internet]. Rio de Janeiro: Instituto Brasileiro de Geografia e Estatística; 2014 [citado 2020 Jan 9]. 181 p. Disponível em: ftp://ftp.ibge.gov.br/PNS/2013/pns2013.pdf

26. Brasil. Ministério da Saúde. Secretaria de Vigilância em Saúde. Departamento de Vigilância de Doenças e Agravos não Transmissíveis e Promoção da Saúde. Vigitel Brasil 2018: vigilância de fatores de risco e proteção para doenças crônicas por inquérito telefônico: estimativas sobre frequência e distribuição sociodemográfica de fatores de risco e proteção para doenças crônicas nas capitais dos 26 estados brasileiros e no Distrito Federal em 2018. Brasília: Ministério da Saúde, 2019, 131 p.

27. Picon RV, Fuchs FD, Moreira LB, Riegel G, Fuchs SC. Trends in prevalence of hypertension in Brazil: a systematic review with meta-analysis. PLoS ONE. 2012;7(10):e48255

28. Chor D, Pinho Ribeiro AL, Sá Carvalho M, Duncan BB, Andrade Lotufo P Araújo Nobre A, et al. Prevalence, Awareness, Treatment and Influence of Socioeconomic Variables on Control of High Blood Pressure: Results of the ELSA-Brasil Study. PLoS ONE. 2015;10(6):e0127382. 
29. International Diabetes Federation. IDF Diabetes Atlas, 9th edn. Brussels, Belgium: 2019. Available at: https://www.diabetesatlas.org

30. Lotufo PA, Santos RD, Figueiredo RM, Pereira AC, Mill JG, Alvim SM, et al. Prevalence, awareness, treatment, and control of high low-density lipoprotein cholesterol in Brazil: Baseline of the Brazilian Longitudinal Study of Adult Health (ELSA-Brasil). J Clin Lipidol. 2016 May-Jun;10(3):568-76.

31. Miranda JJ, Herrera VM, Chirinos JA, Gómez LF, Perel P, Pichardo R, et al. Major cardiovascular risk factors in Latin America: a comparison with the United States. The Latin American Consortium of Studies in Obesity (LASO). PLoS ONE. 2013;8(1): e54056.

32. Ponte-Negretti Cl, Isea-Perez JE, Lorenzatti AJ, Lopez-Jaramillo P, Wyss-Q FS, Pintó X, et al. Atherogenic Dyslipidemia in Latin America: Prevalence, causes and treatment: Expert's position paper made by The Latin American Academy for the Study of Lipids (ALALIP) Endorsed by the Inter-American Society of Cardiology (IASC), the South American Society of Cardiology (SSC), the Pan-American College of Endothelium (PACE), and the International Atherosclerosis Society (IAS). Int J Cardiol. 2017 Sep 15;243:516-22.

33. Rtveladze K, Marsh T, Webber L, Kilpi F, Levy D, Conde W, et al. Health and economic burden of obesity in Brazil. PLoS ONE. 2013;8(7): e68785.

34. Precoma D, Oliveira GMA, Simão AF, Dutra OP, Coelho CR, Izar MCO, Povoa RMD, et al, Sociedade Brasileira de Cardiologia. Atualização da Diretriz de Prevenção Cardiovascular da Sociedade Brasileira de Cardiologia - 2019. Arq Bras Cardiol. 2019;113(4):787-891.

35. Dahrouge S, Kaczorowski J, Dolovich L, Paterson M, Thabane L, Tu K, et al. Long-term outcomes of cluster randomized trial to improve cardiovascular health at population level: The Cardiovascular Health Awareness Program (CHAP). PLoS ONE. 2018;13(9): e0201802.

36. Schmidt MI, Duncan BB, Mill JG, Lotufo PA, Chor D, Barreto SM, et al. Cohort Profile: Longitudinal Study of Adult Health (ELSA-Brasil). IntJ Epidemiol. 2015 Feb;44(1):68-75.

37. Coelho VG, Caetano LF, Liberatore Júnior Rdel R, Cordeiro JA, Souza DR. Perfil lipídico e fatores de risco para doenças cardiovasculares em estudantes de medicina. Arq Bras Cardiol. 2005 Jul;85(1):57-62
38. Faganello LS, Pimentel M, Polanczyk CA, Zimerman T, Malachias MVB, Dutra OP, et al. O Perfil do Cardiologista Brasileiro - Uma Amostra de Sócios da Sociedade Brasileira de Cardiologia. Arq Bras Cardiol. 201906 27;113(1):62-8.

39. Rodríguez-Reyes RR, Navarro-Zarza JE, Tello-Divicino TL, Parra-Rojas I, Zaragoza-García O, Guzmán-Guzmán IP. [Detection of cardiovascular risk in healthcare workers on the basis of WHO/JNC 7/ATP III criteria]. Rev Med Inst Mex Seguro Soc. 2017 May-Jun; 55(3):300-8.

40. Oğuz A, Sağun G, Uzunlulu M, Alpaslan B, Yorulmaz E, Tekiner E, et al. Frequency of abdominal obesity and metabolic syndrome in healthcare workers and their awareness levels about these entities. Turk Kardiyol Dern Ars. 2008 Jul;36(5):302-9.

41. Marochi LH, Campos CW, Marcante FP, Moreira DM. Comparação de fatores de risco cardiovascular entre médicos cardiologistas e não cardiologistas. Rev Bras Cardiol. 2013;26(4):248-52.

42. Mach F, Baigent C, Catapano AL, Koskinas KC, Casula M, Badimon L, et al. 2019 ESC/EAS Guidelines for the management of dyslipidaemias: lipid modification to reduce cardiovascular risk. Eur Heart J. 2020 Jan $1 ; 41(1): 111-88$

43. Appel LJ, Brands MW, Daniels SR, Karanja N, Elmer PJ, Sacks FM. Dietary approaches to prevent and treat hypertension: a scientific statement from the American Heart Association. Hypertension. 2006 Feb;47(2):296-308.

44. Backer G. Epidemiology and prevention of cardiovascular disease: Quo vadis. Eur J Prev Cardiol. 2017 05;24(7):768-72.

45. Cosentino F, Grant PJ, Aboyans V, Bailey CJ, Ceriello A, Delgado V, et al. 2019 ESC Guidelines on diabetes, pre-diabetes, and cardiovascular diseases developed in collaboration with the EASD. Eur Heart J. 2020 Jan 7;41(2):255-323.

46. Schmidt MI, Hoffmann JF, Fátima Sander Diniz M, Lotufo PA, Griep $\mathrm{RH}$, Bensenor IM, et al. High prevalence of diabetes and intermediate hyperglycemia - The Brazilian Longitudinal Study of Adult Health (ELSABrasil). Diabetol Metab Syndr. 2014;6:123. 